1. Heng HH, et al. Stochastic cancer progression driven by non-clonal chromosome aberrations. $J$ Cell Physiol. 2006;208(2):461-472.

2. Magurran AE. Measuring Biological Diversity. Malden, MA: Blackwell Publishing; 2004.

3. Efron B, Thisted R. Estimating the number of unseen species: How many words did Shakespeare know? Biometrika. 1976;63:435-447.

4. de Grouchy J, de Nava C, Cantu JM, Bilski-Pasquier G, Bousser J. Models for clonal evolutions: a study of chronic myelogenous leukemia. Am J Hum Genet. 1966;18(5):485-503

5. Ford CE, Clarke CM. Cytogenetic evidence of clonal proliferation in primary reticular neoplasms. Can Cancer Conf. 1963;5:129-146.

6. Dawe CJ. Phylogeny and oncogeny. Natl Cancer Inst Monogr. 1969;31:1-40.

7. Yosida TH. Relation between chromosomal alteration and development of tumors. Jpn J Genet. 1966:41:439-451.

8. Siegmund KD, Marjoram P, Woo YJ, Tavare S, Shibata D. Inferring clonal expansion and cancer stem cell dynamics from DNA methylation patterns in colorectal cancers. Proc Natl Acad Sci US A. 2009;106(12):4828-4833.

9. Tsao JL, Tavare S, Salovaara R, Jass JR, Aaltonen LA, Shibata D. Colorectal adenoma and cancer divergence. Evidence of multilineage progression. Am J Pathol. 1999;154(6):1815-1824.

10. Maley CC, et al. Genetic clonal diversity predicts progression to esophageal adenocarcinoma. Nat Genet. 2006;38(4):468-473.

11. Park SY, Gönen M, Kim HJ, Michor F, Polyak K. Cellular and genetic diversity in the progression of in situ human breast carcinomas to an invasive phenotype. J Clin Invest. 2010;120(2):636-644.

12. Galipeau PC, et al. NSAIDs modulate CDKN2A, TP53, and DNA content risk for progression to esophageal adenocarcinoma. PLoS Med. 2007;4:e67.

13. Ludwig JA, Weinstein JN. Biomarkers in cancer staging, prognosis and treatment selection. Nat Rev Cancer. 2005;5(11):845-856

14. Pepper J, Findlay CS, Kassen R, Spencer S, Maley C. Cancer research meets evolutionary biology. Evolu- tionary Applications. 2009;2(1):62-70. doi:10.1111/ j.1752-4571.2008.00063.

15. Shipitsin M, et al. Molecular definition of breast tumor heterogeneity. Cancer Cell. 2007;11(3):259-273.

16. Quintana E, Shackleton M, Sabel MS, Fullen DR, Johnson TM, Morrison SJ. Efficient tumour formation by single human melanoma cells. Nature. 2008; 456(7222):593-598

17. Hu M, Polyak K. Microenvironmental regulation of cancer development. Curr Opin Genet Dev. 2008;18(1):27-34.

18. Polyak K, Weinberg RA. Transitions between epithelial and mesenchymal states: acquisition of malignant and stem cell traits. Nat Rev Cancer. 2009; 9(4):265-273.

19. Roche-Lestienne C, Preudhomme C. Mutations in the ABL kinase domain pre-exist the onset of imatinib treatment. Semin Hematol. 2003;40(2 Suppl 2):80-82.

20. Shah NP, et al. Sequential ABL kinase inhibitor therapy selects for compound drug-resistant BCRABL mutations with altered oncogenic potency. J Clin Invest. 2007;117(9):2562-2569.

\title{
TGF- $\beta$ in the pathogenesis and prevention of disease: a matter of aneurysmic proportions
}

\author{
Harry C. Dietz
}

Institute of Genetic Medicine and Howard Hughes Medical Institute, Johns Hopkins University School of Medicine, Baltimore, Maryland, USA.

TGF- $\beta$ regulates many aspects of cellular performance relevant to tissue morphogenesis and homeostasis. Postnatal perturbation of TGF- $\beta$ signaling contributes to the pathogenesis of many disease states, as recently exemplified through the study of Marfan syndrome (MFS), including aortic aneurysm and skeletal muscle myopathy. Heterogeneity in the regulation and consequences of TGF- $\beta$ signaling, amplified in the context of disease, has engendered confusion and controversy regarding its utility as a therapeutic target. Three studies recently published in the JCI, including one in this issue, underscore the complexity of this subject. Heydemann and colleagues implicate dimorphic variation in latent TGF- $\beta$-binding protein 4 (LTBP4), a regulator of TGF- $\beta$ bioavailability and activation, as a modifier of muscular dystrophy in $\gamma$-sarcoglycan-deficient mice. In contrast to experience with ascending aortic aneurysm in MFS, Wang and colleagues show that systemic abrogation of TGF- $\beta$ signaling worsens (rather than attenuates) Ang II-induced abdominal aortic aneurysm progression in mice. Tieu and colleagues define alterations in the regulation of vascular inflammation in the pathogenesis of Ang II-induced aneurysm and dissection in mice, which may help shed some light on this apparent paradox.

Historically, perturbation (largely enhancement) of TGF- $\beta$ signaling has been strongly implicated in the pathogenesis of diverse disease states, prominently including the initiation and progression of cancer and tissue fibrosis. Despite decades of intensive study in these contexts, the net effects

Conflict of interest: The author has declared that no conflict of interest exists.

Citation for this article: JClin Invest. 2010; 120(2):403-407. doi:10.1172/JCI42014 of TGF- $\beta$ signaling in disease pathogenesis and, perhaps more importantly, the incorporation of its antagonism into therapeutic strategies, remain controversial. This is nicely illustrated by the so-called TGF- $\beta$ cancer paradox. In brief, TGF- $\beta$ plays a prominent role in the suppression of tumorigenesis through induction of cellcycle arrest and apoptosis and maintenance of cellular differentiation, as evidenced by the frequent biallelic loss of genes encoding TGF- $\beta$ receptors or intracellular media- tors of signaling in multiple tumor types (for example, see ref. 1). Attenuation or loss of tumor responsiveness can induce upregulation of TGF- $\beta$ ligand expression, resulting in excessive stimulation of the signaling-intact neighboring stroma (reviewed in ref. 2). Attributable consequences include impairment of tumor surveillance through inhibition of adaptive immunity, acceleration of tumor growth through enhancement of angiogenesis, and induction of tumor invasion and metastasis through stimulation of innate immunity (including mast cell, monocyte, and macrophage chemotaxis), compromising of endothelial boundaries, and promotion of epithelial- or endothelialmesenchymal transition (EpMT [ref. 2] and EnMT [ref. 3], respectively; collectively EMT). Amplification of TGF- $\beta$ signaling can occur due to enhanced TGF- $\beta$ ligand expression (e.g., by recruited mast cells or macrophages) or activation (e.g., by MMPs or simple fibrosis-dependent enhancement of shear stress within the tumor microenvironment; ref. 4). Additional TGF- $\beta$-related paradoxes are evident. For example, TGF- $\beta$ can either induce or suppress angiogenesis depending upon its concentration and repertoire of TGF- $\beta$ receptors and accessory proteins (2). 


\section{TGF- $\beta$ in Marfan syndrome and related disorders}

Insights gained while studying Marfan syndrome (MFS), a systemic connective tissue disorder caused by deficiency of the microfibrillar protein fibrillin-1, highlighted the potential role of TGF- $\beta$ in the pathogenesis of many additional disease phenotypes. Manifestations include ocular lens dislocation, a strong predisposition for aortic root enlargement and dissection, skeletal overgrowth, pulmonary emphysema, myxomatous valve disease, and skeletal muscle hypoplasia and myopathy (reviewed in ref. 5). In contrast to early pathogenetic models in which tissue weakness alone was invoked, a fibrillin-1-deficient mouse model showed evidence for enhanced TGF- $\beta$ signaling in most affected tissues. The fibrillins interact directly with the latent TGF- $\beta$-binding protein (LTBP) component of the large latent complex (LLC) of TGF- $\beta$ that includes the mature cytokine, a dimer of its processed $\mathrm{N}$-terminal propeptide termed latencyassociated peptide (LAP), and one of three LTBP isoforms (LTBP-1, -3, or -4). Current theory posits that microfibrils composed of fibrillin-1 sequester the LLC upon its secretion and limit accessibility to or the efficiency of activators of TGF- $\beta$. In this view, fibrillin-1 deficiency would result in failed matrix sequestration and hence promiscuous activation of and signaling by TGF- $\beta$. Importantly, many critical phenotypes could be attenuated or prevented in Marfan mouse models by systemic delivery of pan-specific TGF- $\beta$-neutralizing antibody (TGF $\beta N A b)$, including developmental emphysema (6), myxomatous degeneration of the mitral valve (7), aortic root aneurysm (8), and skeletal myopathy (9). The apparent mechanism of rescue varied by tissue type. TGF- $\beta$ antagonism attenuated apoptosis of septal progenitors in the lung but enhanced apoptosis and suppressed cellular proliferation in the mitral valve. Improvement in skeletal muscle mass, architecture, and function correlated with decreased muscle fibrosis and enhanced regeneration after induced injury. Importantly, these protective effects were mimicked by treatment of Marfan mice with losartan, an FDA-approved Ang II type 1 receptor (AT1R) blocker (ARB) that can limit TGF- $\beta$ signaling through suppression of expression of TGF- $\beta$ ligands, receptors, and activators such as thrombospondin-1 and MMPs. Indeed, losartan-induced phenotypic improvement in each tissue correlated with direct in vivo evidence for
TGF- $\beta$ antagonism in Marfan mice $(8,9)$. Apparent protection against aneurysm progression by losartan was also seen in a small observational cohort of children with severe MFS (10), supporting the launch of a multicenter clinical trial (11).

Experience with other patient cohorts and experimental systems has engendered both confidence and ambiguity regarding the role of TGF- $\beta$ in the pathogenesis of aortic aneurysm. Meaningful evidence for increased TGF- $\beta$ signaling in the vessel wall has been observed in patients and/or mice with recessive cutis laxa with aneurysm (caused by deficiency of fibulin-4) (12), arterial tortuosity syndrome (deficiency of GLUT10) (13), and bicuspid aortic valve with aneurysm (etiology largely unknown) (14). Perhaps most convincingly, primary heterozygous mutations in the TGF- $\beta$ receptor subunits (ALK5 or T $\beta R 2$ ) were found to underlie Loeys-Dietz syndrome (LDS) and other Marfan-like phenotypes that show a strong predisposition for aneurysms and dissections throughout the arterial tree, prominently including the aortic root (15-17). Nearly all the mutations are missense substitutions within the kinase domain of either receptor subunit. While this mutational repertoire argues against a haploinsufficiency mechanism, it is notable that recombinant expression of mutant receptors in cells naive for the corresponding receptor subtype failed to support TGF- $\beta$ signaling (17). However, by mechanisms that remain to be elucidated, patient vascular tissue obtained at surgery or autopsy has consistently shown increased TGF- $\beta$ signaling (nuclear accumulation of phospho-Smad2/3 [pSmad-2/3] and increased output of TGF- $\beta$-responsive genes) $(15,16,18)$. The paradox is widened by the observation that several LDS phenotypes (e.g., craniosynostosis and cleft palate) have been associated with TGF- $\beta$ deficiency states in mice (discussed in ref. 16). Furthermore, lineage-specific ablation of TGF- $\beta$ signaling in vascular smooth muscle cells resulted in alterations in vascular morphogenesis in mice, including impaired elastogenesis and vessel widening (19). While interesting, the relevance of these results to postnatal presentations of aneurysm is difficult to assess due to associated cardiovascular malformations and early prenatal mortality.

\section{TGF- $\beta$ and muscular dystrophy}

Based upon observations made in Marfan mouse models, it was postulated that
TGF- $\beta$ might contribute to failed compensation in Duchenne muscular dystrophy (DMD), which is caused by a deficiency of the muscle structural protein dystrophin and characterized by muscle fiber fragility. Boys with DMD initially do well while their pace of muscle regeneration matches that of destruction. Later in childhood they show a rapid clinical decline that correlates with increased fibrosis and waning regenerative capacity, events plausibly mediated by TGF- $\beta$. The $m d x$ mouse model of DMD shows sustained elevation of TGF- $\beta$ signaling in muscle, and postnatal treatment with either TGF $\beta \mathrm{NAb}$ or losartan limited injuryinduced fibrosis and maintained muscle regeneration, architecture, and function (9). There was no corresponding decrease in creatine kinase levels, suggesting that therapy did not address fiber fragility, but rather specifically enhanced repair.

In a recent issue of the JCI, Heydemann and colleagues used an unbiased mapping approach to identify genetic modifiers of the muscle phenotype in $\gamma$-sarcoglycandeficient (Sgcg-deficient) mice, a model of limb girdle muscular dystrophy type $2 \mathrm{C}$ (20). Targeted disruption of Sgcg resulted in more severe disease in the DBA/2J (D2) strain when compared with the 129T2/ SvEmsJ strain (129 strain) using markers of membrane leak and fibrosis as outcome measures. Remarkably, it was shown that the variation modifying both phenotypes localized to a single locus on chromosome 7 that harbors the genes encoding both TGF- $\beta 1$ and LTBP4, among many others. While no sequence variation was observed in $T g f b 1$, there was an insertion/deletion (indel) dimorphism in the coding sequence of $L t b p 4$ resulting in the loss of 12 amino acids in the predisposed (D2) form of the protein within a domain that is cleaved by selected activators of TGF- $\beta$. Fibroblasts derived from D2 mice showed an increased ratio of cleaved to uncleaved LTBP4 when exposed to either pancreatin or plasmin, as compared with the 129 strain, as did a D2 recombinant LTBP4 peptide encompassing the indel. D2 fibroblasts (with or without Sgcg targeting) also showed increased phosphorylation of Smad2 when treated with recombinant TGF- $\beta$ compared with the 129 strain, and there was evidence for increased nuclear accumulation of $\mathrm{pSmad} 2$ within the muscle fibers of D2-Sgcg-targeted mice when compared with mutant 129 animals. While these findings are provocative, a number of limitations preclude definitive conclusion that variation 
in LTBP4 or TGF- $\beta$ signaling govern phenotypic modification in this mouse model. First, while the ratio of cleaved to uncleaved LTBP4 was increased in the D2 strain, the amount of cleaved LTBP4 varied widely within a given background and did not differ between strains. The amount of TGF- $\beta$ signaling should correlate with the amount of cleaved LTBP4 and not the ratio. Second, there was little if any cleaved LTBP4 in either D2 or 129 fibroblasts that were not treated with exogenous enzyme, and no difference between the strains. Given that TGF- $\beta$ secretion, matrix sequestration, and activation occurs in culture (with physiologic activators), this suggests that the predisposition for cleavage imposed by the LTBP4 deletion is modest, at best. Third, the higher level of pSmad2 in D2 cells treated with TGF- $\beta$ is hard to interpret despite the use of a recombinant form that is associated with LAP (small latent complex [SLC]). There is no evidence that the exogenous SLC interacts with matrix-incorporated LTBP4 in culture (most TGF- $\beta$ is secreted from the cell in the context of the LLC, with the LTBP-LAP interaction established intracellularly). Furthermore, the SLC can be directly activated without ever associating with LTBPs by many physiologic activators, including thrombospondin-1 and plasmin. Without knowing the molar ratio of LTBP4 and SLC in this experiment, or that they interact, it is impossible to know whether LTBP4 has anything to do with this result. Finally, the increased nuclear localization of pSmad2 in D2-Sgcg-targeted muscle was largely restricted to newly regenerated fibers with central nucleation. This raises the question of whether high TGF- $\beta$ signaling is causing injury or simply a marker of recent injury in this model. Additional experiments might include correlation of severe disease with the deleted Ltbp4 allele in other backgrounds and the use of genetic and/or pharmacologic strategies to determine whether TGF- $\beta$ antagonism levels the playing field between D2 and $129 \gamma$-sarcoglycan-deficient mice. Given that validation of the conclusions of this high-quality study would both consolidate and extend current thinking regarding the potential utility of TGF- $\beta$ antagonists in myopathic states, it is certainly worth the effort.

\section{TGF- $\beta$ and models}

\section{of abdominal aortic aneurysm}

Abdominal aortic aneurysm (AAA) is a major cause of morbidity and mortality.
Late lesions are characterized by intense matrix degradation in the setting of severe inflammation. While there is a familial predisposition, the relevant genes are unknown, and mouse models typically involve mechanical or chemical injury. Infusion of Ang II is known to induce inflammatory aneurysms and dissections of the suprarenal abdominal aorta and ascending aorta in mice, with reduced frequency and severity in the latter. Disease is greatly accentuated in Ang II-infused Apoe - $^{--}$mice via a poorly understood mechanism that appears to be independent of atherosclerosis or hypertension (21). In this issue of the JCI, Wang and colleagues show that treatment of normocholesterolemic C57BL/ 6 mice with TGF $\beta$ NAb breaks their resistance to Ang II-induced AAA and dissection, with both a greater frequency and severity of lesions and accelerated death (22). Relative (but not complete) improvement in incidence, severity, and/or survival was seen after manipulations focused on modulation of either the adaptive or innate immune response, including depletion or functional impairment of T cells, B cells, or monocytes and silencing of MMP12 (macrophage elastase) expression. On this basis, the authors caution against extrapolating treatment strategies for MFS to other presentations of aneurysm. While inherently true, independent of findings in this study, can this recommendation be refined? The lesions observed in TGF $\beta$ NAb-treated and Ang II-infused C57BL/6 mice were similar to those in other Ang II infusion-based rodent models, including an intense infiltration of macrophages and lymphocytes that begins in the adventitia and elastolysis (albeit focal and patchy) within the media. Remarkably, inflammation and aneurysm formation are observed within days of infusion, with death due to dissection occurring within days to a few weeks. On this basis, it seems reasonable to hypothesize that antagonists of TGF- $\beta$, a known potent antiinflammatory cytokine, should be particularly avoided in hyperacute inflammatory aneurysms (although a human correlate to this presentation is difficult to imagine). Even this may need to be qualified. While Wang and colleagues concede that another study failed to see worsening of aneurysms in TGF $\beta$ NAb-treated Ang II-infused mice, they do not comment upon the fact that this study found that TGF $\beta$ NAb afforded significant protection from Ang II-induced inflammatory aneurysms after Cxcl10 targeting - an event that accentuated aneu- rysm incidence and severity and death (23). It should also be noted that ARBs or silencing of expression of AT1R have been definitively shown to prevent Ang II-induced aneurysm in rodent models (24-28). If such manipulations attenuate TGF- $\beta$ signaling in these models as in MFS (which remains to be demonstrated), then this would suggest that TGF- $\beta$ antagonism is only nonproductive in the setting of (artificially?) intense stimulation of AT1R.

In another recent issue of the JCI, Tieu and colleagues reveal a mechanism by which Ang II induces aortic aneurysms in mice (29). The model involved Ang II infusion into aged C57BL/6J mice (without TGF $\beta$ NAb treatment). Previous work had shown increased expression and a pathogenetic role for monocyte chemoattractant protein-1 (MCP-1), its receptor (CCR2), and IL-6 in Ang II-induced vascular inflammation and aneurysm progression (references in ref. 29). It was also known that IL-6 expression was dependent, at least in part, on CCR2 signaling (30). Tieu and colleagues show that Ang II infusion induces the adventitial accumulation of CCR2 $2^{+}$ macrophages, with specific enrichment of activated macrophages at sites of dissection. CCR2-null animals showed reduced accumulation of macrophages, IL- 6 and MCP-1 expression, and incidence of dissection in response to Ang II; the latter two effects were also observed in IL-6-null mice. Interestingly, Ang II induced the recruitment of infused CCR2 $2^{+/+}$monocytes to both the ascending and suprarenal descending aorta in CCR2-null animals. This resulted in increased expression of IL- 6 and MCP-1 and increased dissection (when compared with infusion of CCR2 $2^{-/}$monocytes). While these data demonstrate that IL-6 and MCP-1 contribute to Ang II-induced aneurysm and that recruited CCR $2^{+}$monocytes themselves serve to amplify the inflammatory cascade, perhaps the most novel aspect of this study was the demonstration that coculture of monocytes with adventitial fibroblasts resulted in their enhanced expression of IL-6 and IL-6-dependent macrophage differentiation/enhanced MCP-1 expression; this required an unspecified paracrine factor. Notably, while CCR2 or IL-6 silencing prevented early dissection, this did not prevent later dissection with chronic Ang II infusion. It is also notable that while human aneurysm tissue showed increased IL- 6 expression in the adventitia, this was restricted to zones of dissection. These data suggest that while the described 
IL-6/MCP-1 amplification loop contributes to the predisposition for Ang II-induced aneurysm and dissection in mice, it is not required and may either be a marker of dissection-induced inflammation or have a restricted role late in disease in people.

While Tieu and colleagues (29) do not speculate upon the nature of the fibroblast-derived paracrine factor that stimulates monocyte cytokine expression and macrophage differentiation, it is interesting to speculate that TGF- $\beta$ may play a role. Indeed, TGF- $\beta$ is known to induce IL- 6 and/or MCP-1 expression in many cell types, including fibroblasts and vascular smooth muscle cells (31-35) and to induce innate immune responses, including monocyte recruitment and macrophage differentiation. These events can stimulate myofibroblast formation from fibroblasts or endothelial cells (EnMT), which in turn may amplify the process through secretion of TGF- $\beta$, MMPs, or even MCP-1 $(36,37)$ in some tissues. Furthermore, the combination of IL- 6 and TGF- $\beta$ can induce the production of Th 17 cells from either naive $\mathrm{CD} 4^{+} \mathrm{T}$ helper cells or Tregs, an event that induces an extreme proinflammatory environment (38).

\section{Perspective}

Taken together, the studies discussed in this commentary highlight the complex and context-dependent roles of TGF- $\beta$ in disease. From a therapeutic standpoint, it seems essential to define critical events in disease pathogenesis, both upstream and downstream of TGF- $\beta$, in order to avoid getting lost in the noise. This may best be accomplished using genetically defined animal models that closely replicate the disease mechanism and the physiologic complexity of the human condition. It also seems essential to recognize that the role of TGF- $\beta$ may vary during the dynamic transition from predisposition to terminal events, as nicely exemplified by the TGF- $\beta$ cancer paradox. While current data support the conclusion that TGF- $\beta$ contributes to aneurysm progression in MFS, it is possible that reduced TGF- $\beta$ signaling contributes to predisposition (given that fibrillins are also needed to concentrate TGF- $\beta$ superfamily members and support signaling at sites of intended function) or that enhanced signaling is protective in the setting of late inflammation or during wound healing after dissection. This potential pathogenetic sequence is even more intuitive for LDS. The studies by Wang et al. (22) and Tieu et al. (29) both emphasize events in the adventitia during aneurysm formation, in contrast to the historical focus on the media prompted by the near-uniform finding of elastolysis in this compartment. Has this been a distraction? The old observation of maintenance of wall integrity after endarterectomy (in essence, stripping of the entire media) and the relatively new observation that fibulin-5-deficient mice and people do not get aneurysms despite failed elastogenesis and generalized medial disorganization (39-41) should provoke a challenge to long-held assumptions and focus attention on the adventitia. The study by Heydemann and colleagues (20) highlights the need for consideration of individual variation in the development of pathogenetic models or in the design and interpretation of clinical trials. It should also be noted that the TGF- $\beta$ family (and superfamily) comprises cytokines with diverse and often opposing functions. A colleague told me recently that the number of times the word "context" is used while discussing TGF- $\beta$ is inversely proportional to the speaker's understanding. If this is true, I profess my ignorance.

Address correspondence to: Harry C. Dietz, Institute of Genetic Medicine, Johns Hopkins University School of Medicine, BRB 539,733 N. Broadway, Baltimore, Maryland 21205, USA. Phone: (410) 614-0701; Fax: (410) 614-2256; E-mail: hdietz@jhmi.edu.

1. Markowitz S, et al. Inactivation of the type II TGFbeta receptor in colon cancer cells with microsatellite instability. Science. 1995;268(5215):1336-1338.

2. Tian M, Schiemann WP. The TGF-beta paradox in human cancer: an update. Future Oncol. 2009;5(2):259-271.

3. Potenta S, Zeisberg E, Kalluri R. The role of endothelial-to-mesenchymal transition in cancer progression. Br J Cancer. 2008;99(9):1375-1379.

4. Wipff PJ, Rifkin DB, Meister JJ, Hinz B. Myofibroblast contraction activates latent TGFbeta 1 from the extracellular matrix. J Cell Biol. 2007;179(6):1311-1323.

5. Ramirez F, Dietz HC. Marfan syndrome: from molecular pathogenesis to clinical treatment. Curr Opin Genet Dev. 2007;17(3):252-258.

6. Neptune ER, et al. Dysregulation of TGF-beta activation contributes to pathogenesis in Marfan syndrome. Nat Genet. 2003;33(3):407-411.

7. $\mathrm{Ng} \mathrm{CM}$, et al. TGF-beta-dependent pathogenesis of mitral valve prolapse in a mouse model of Marfan syndrome. J Clin Invest. 2004;114(11):1586-1592.

8. Habashi JP, et al. Losartan, an AT1 antagonist, prevents aortic aneurysm in a mouse model of Marfan syndrome. Science. 2006;312(5770):117-121.

9. Cohn RD, et al. Angiotensin II type 1 receptor blockade attenuates TGF-beta-induced failure of muscle regeneration in multiple myopathic states. Nat Med. 2007;13(2):204-210.

10. Brooke BS, Habashi JP, Judge DP, Patel N, Loeys B, Dietz HC 3rd. Angiotensin II blockade and aortic- root dilation in Marfan's syndrome. N Engl J Med. 2008;358(26):2787-2795.

11. Lacro RV, et al. Rationale and design of a randomized clinical trial of beta-blocker therapy (atenolol) versus angiotensin II receptor blocker therapy (losartan) in individuals with Marfan syndrome. Am Heart J. 2007;154(4):624-631.

12. Hanada K, et al. Perturbations of vascular homeostasis and aortic valve abnormalities in fibulin-4 deficient mice. Circ Res. 2007;100(5):738-746.

13. Coucke PJ, et al. Mutations in the facilitative glucose transporter GLUT10 alter angiogenesis and cause arterial tortuosity syndrome. Nat Genet. 2006;38(4):452-457.

14. Gomez D, et al. Syndromic and non-syndromic aneurysms of the human ascending aorta share activation of the Smad2 pathway. J Pathol. 2009; 218(1):131-142.

15. Loeys BL, et al. Aneurysm syndromes caused by mutations in the TGF-beta receptor. $N$ Engl J Med. 2006;355(8):788-798.

16. Loeys BL, et al. A syndrome of altered cardiovascular, craniofacial, neurocognitive and skeletal development caused by mutations in TGFBR1 or TGFBR2. Nat Genet. 2005;37(3):275-281.

17. Mizuguchi T, et al. Heterozygous TGFBR2 mutations in Marfan syndrome. Nat Genet. 2004;36(8):855-860.

18. Maleszewski JJ, Miller DV, Lu J, Dietz HC, Halushka MK. Histopathologic findings in ascending aortas from individuals with Loeys-Dietz syndrome (LDS). Am J Surg Pathol. 2009;33(2):194-201.

19. Choudhary B, Zhou J, Li P, Thomas S, Kaartinen V, Sucov HM. Absence of TGFbeta signaling in embryonic vascular smooth muscle leads to reduced lysyl oxidase expression, impaired elastogenesis, and aneurysm. Genesis. 2009;47(2):115-121.

20. Heydemann A, et al. Latent TGF- $\beta$-binding protein 4 modifies muscular dystrophy in mice. J Clin Invest. 2009;119(12):3703-3712.

21. Cassis LA, et al. ANG II infusion promotes abdominal aortic aneurysms independent of increased blood pressure in hypercholesterolemic mice. Am J Physiol Heart Circ Physiol. 2009;296(5):H1660-H1665.

22 . Wang $Y$, et al. TGF- $\beta$ activity protects against inflammatory aortic aneurysm progression and complications in angiotensin II-infused mice.J Clin Invest. 2010;120(2):422-432.

23. King VL, et al. Interferon-gamma and the interferon-inducible chemokine CXCL10 protect against aneurysm formation and rupture. Circulation. 2009; 119(3):426-435.

24. Daugherty A, Manning MW, Cassis LA. Antagonism of AT2 receptors augments angiotensin IIinduced abdominal aortic aneurysms and atherosclerosis. Br J Pharmacol. 2001;134(4):865-870.

25. Cassis LA, Rateri DL, Lu H, Daugherty A. Bone marrow transplantation reveals that recipient AT1a receptors are required to initiate angiotensin II-induced atherosclerosis and aneurysms. Arterioscler Thromb Vasc Biol. 2007;27(2):380-386.

26. Henriques T, Zhang X, Yiannikouris FB, Daugherty A, Cassis LA. Androgen increases AT1a receptor expression in abdominal aortas to promote angiotensin II-induced AAAs in apolipoprotein E-deficient mice. Arterioscler Thromb Vasc Biol. 2008; 28(7):1251-1256.

27. Fujiwara $Y$, et al. Inhibition of experimental abdominal aortic aneurysm in a rat model by the angiotensin receptor blocker valsartan. Int J Mol Med. 2008;22(6):703-708.

28. Inoue $\mathrm{N}$, et al. Involvement of vascular angiotensin II-forming enzymes in the progression of aortic abdominal aneurysms in angiotensin II- infused ApoE-deficient mice. J Atheroscler Thromb. 2009; 16(3):164-171.

29. Tieu BC, et al. An adventitial IL-6/MCP1 amplification loop accelerates macrophage-mediated vascular inflammation leading to aortic dissection in 
mice. J Clin Invest. 2009;119(12):3637-3651.

30. Ishibashi $\mathrm{M}$, et al. Bone marrow-derived monocyte chemoattractant protein-1 receptor CCR2 is critical in angiotensin II-induced acceleration of atherosclerosis and aneurysm formation in hypercholesterolemic mice. Arterioscler Thromb Vasc Biol. 2004;24(11):e174-178.

31. Lee EY, et al. The monocyte chemoattractant protein-1/CCR2 loop, inducible by TGF-beta, increases podocyte motility and albumin permeability. AmJ Physiol Renal Physiol. 2009;297(1):F85-F94.

32. Park JI, et al. Transforming growth factor-beta 1 activates interleukin- 6 expression in prostate cancer cells through the synergistic collaboration of the Smad2, p38-NF-kappaB, JNK, and Ras signaling pathways. Oncogene. 2003;22(28):4314-4332.

33. Seong GJ, et al. TGF-beta-induced interleukin-6 participates in transdifferentiation of human Ten- on's fibroblasts to myofibroblasts. Mol Vis. 2009; 15:2123-2128.

34. Zhang F, et al. Transforming growth factor-beta promotes recruitment of bone marrow cells and bone marrow-derived mesenchymal stem cells through stimulation of MCP-1 production in vascular smooth muscle cells. J Biol Chem. 2009;284(26):17564-17574.

35 . Eickelberg O, et al. Transforming growth factorbeta 1 induces interleukin- 6 expression via activating protein-1 consisting of JunD homodimers in primary human lung fibroblasts.J Biol Chem. 1999; 274(18):12933-12938.

36. Dagouassat $\mathrm{M}$, et al. Monocyte chemoattractant protein-1 (MCP-1)/CCL2 secreted by hepatic myofibroblasts promotes migration and invasion of human hepatoma cells. Int J Cancer. 2010;126(5):1095-1108. 37. Pinchuk IV, et al. Monocyte chemoattractant protein-1 production by intestinal myofibroblasts in response to staphylococcal enterotoxin a: relevance to staphylococcal enterotoxigenic disease. J Immunol. 2007;178(12):8097-8106.

38. Afzali B, Mitchell P, Lechler RI, John S, Lombardi G. 2009. Translational Mini-Review Series on Th17 Cells: induction of interleukin-17 production by regulatory T cells. Clin Exp Immunol. [published online ahead of print Nov 11 2009]. doi: 10.1111/ j.1365-2249.2009.04038.x.

39. Yanagisawa $\mathrm{H}$, et al. Fibulin-5 is an elastin-binding protein essential for elastic fibre development in vivo. Nature. 2002;415(6868):168-171.

40. Nakamura T, et al. Fibulin-5/DANCE is essential for elastogenesis in vivo. Nature. 2002; 415(6868):171-175.

41. Loeys B, et al. Homozygosity for a missense mutation in fibulin-5 (FBLN5) results in a severe form of cutis laxa. Hum Mol Genet. 2002;11(18):2113-2118. 